\title{
Optimized production of a high-performance hybrid biomaterial: biomineralized spider silk for bone tissue engineering
}

\author{
Alessandra Dellaquila (D), ${ }^{1}$ Gabriele Greco \\ Barbara Mazzolai, ${ }^{3}$ Anna Tampieri
}

\author{
,2,3 Elisabetta Campodoni \\ ,${ }^{1}$ Nicola M. Pugno (D), 2,4,5 Monica Sandri
${ }^{1}$ Mauro Mazzocchi, ${ }^{1}$ (i) 1

${ }_{1}^{1}$ ISTEC CNR - Institute of Science and Technology for Ceramics, National Research Council, Via Granarolo 64, Faenza 48018, Italy 2 Laboratory of Bio-inspired \& Graphene Nanomechanics, Department of Civil, Environmental and Mechanical Engineering, University of Trento, Via Mesiano 77, Trento 38123 , Italy 3 Center for Micro-BioRobotics@SSSA, Istituto Italiano di Tecnologia, Viale Rinaldo Piaggio 34, Pontedera I-56025, Italy 4 School of Engineering and Materials Science, Queen Mary University of London, Mile End Road, E1 4NS, London, United Kingdom 5 Ket-Lab, Edoardo Amaldi Foundation, Via del Politecnico snc, Rome 00133, Italy

Correspondence to: M. Sandri (E-mail: monica.sandri@istec.cnr.it) and N. M. Pugno (E-mail: nicola.pugno@unitn.it)

ABSTRACT: Silks have been widely used as biomaterials due to their biocompatibility, biodegradability, and excellent mechanical properties. In the present work, native spider silk was used as organic template for controlled nucleation of hydroxyapatite (HA) nano-coating that can act as biomimetic interface. Different bio-inspired neutralization methods and process parameters were evaluated to optimize the silk functionalization. The morphology and chemical composition were investigated by scanning electron microscopy, energydispersive X-ray spectroscopy, Fourier transform infrared spectroscopy, and X-ray diffraction analysis and mechanical properties were studied through tensile tests. Results showed that the optimized protocol enabled a controlled and homogeneous nucleation of apatite nano-crystals while maintaining silk mechanical performances after mineralization. This study reports the optimization of a simple and effective bio-inspired nucleation process for precise nucleation of HA onto spider silk templates, suitable to develop high-performance hybrid interfaces for bone tissue engineering. (C) 2019 Wiley Periodicals, Inc. J. Appl. Polym. Sci. 2019, 137, 48739.

KEYWORDS: biomimetic; biopolymers and renewable polymers; mechanical properties; surfaces and interfaces

Received 21 June 2019; accepted 4 November 2019

DOI: 10.1002/app.48739

INTRODUCTION

Silks are fibrous protein polymers spun by some species of Lepidoptera (as butterflies and silkworms) and Arachnida, that is, spiders. ${ }^{1}$ These biological polymers have been extensively studied in the past decades because of its biocompatibility, biodegradability, nontoxicity, and excellent mechanical properties, such as tensile strength and toughness. ${ }^{2-4}$

Silk from silkworms, usually fibroin from Bombix mori, has been employed as suture material for centuries, ${ }^{5}$ and it is currently the main silk type used in biomedical field due to its availability and ease of commercial production. ${ }^{2}$ Silk-based biomaterials are used for tissue engineering (TE) applications in form of scaffolds, ${ }^{6}$ hydrogels, ${ }^{7}$ films or fibers, ${ }^{8}$ as well as nanoparticles in drug delivery systems. ${ }^{9,10}$ Moreover, silk-based devices have been successfully designed and developed for regeneration of different tissues, such as bone, tendon, ligament, skin, and nerve. ${ }^{11} \mathrm{Li}$ et al. developed silk fibroin-based scaffolds incorporating nanoparticles of hydroxyapatite (HA) and bone morphogenic protein 2 by electrospinning of silk fibers for bone TE. ${ }^{12}$ Their results revealed an improved bone formation and osteogenesis process in vitro. Shi et al. produced knitted meshes of silk functionalized with low crystallinity HA for regeneration of the bone/ligament interface, demonstrating cell proliferation and differentiation in vitro and recovery of mechanical strength and tissue regeneration in vivo. ${ }^{13}$ 
Spider silk, particularly draglines spun by major ampullate gland, has become of great interest in bio-engineering in the past decades due to the outstanding mechanical properties, higher biocompatibility, and lower immunogenicity compared to silkworm silk. ${ }^{14-16}$ Nevertheless, native spider silk use is limited because of low production yields and difficulty in spiders farming. ${ }^{8,17}$ A feasible solution is to produce engineered synthetic genes and recombinant proteins encoding spider silk. Hardy et al. ${ }^{18}$ developed films made of mineralized biodegradable polyesters and recombinant spider silk proteins [eADF4(C16)], capable to enhance bone tissue formation in vitro. Although the

encouraging results from genetic engineering, ${ }^{19,20}$ a close mimicry of the complex structure and mechanical behavior of spider silk remains an ambitious target.

Alessandra Dellaquila and Gabriele Greco contributed equally to this article.

(C) 2019 Wiley Periodicals, Inc.

Chemical, biological, and mechanical properties ensure that silks can be successfully used in bone TE; furthermore, it represents a suitable template for HA nucleation through biomineralization processes, enhancing bone formation and osteointegration. ${ }^{21-23}$ The mechanism of bone biomineralization consists of simultaneous nucleation of nanoHA crystals on self-assembling collagen matrix, and it has been replicated in vitro successfully. ${ }^{24}$ Other routes ${ }^{25,26}$ for the mimesis of this natural process are based on the use of proteins, natural or synthetic polymers, or organoapatites, ${ }^{27}$ as well as organic templates, such as silks, ${ }^{12}$ while the inorganic phase (HA) is usually synthesized from saltmediated reactions, acid-base (neutralization) syntheses, or by using synthetic body fluids. ${ }^{28}$ Cao et al. ${ }^{29}$ mineralized spider dragline silks through immersion in a HA precursor solution for up to 10 days, demonstrating the possibility of nucleating HA crystals with a preferred orientation. Dmitrovic et al.$^{30}$ produced spider silk coated with calcite via a salt-mediated biomineralization process from calcium chloride and ammonium carbonate. Their data revealed formation of regular rhombic crystals along the fibers, with a potential application as bone grafts.

In this study, bio-inspired HA neutralization synthesis has been optimized to effectively functionalize native silk draglines from Cupiennius salei (Keyserling 1877). As a first step, the effect of reagent concentration, reagents pouring order, and HA maturation time was investigated to obtain a controlled nucleation of inorganic phase. The mineralized silks were then evaluated in terms of coating morphology, chemical composition, and mechanical properties, and results were compared to untreated and supercontracted silks and to HA crystal deposition from a precursor solution. The goal of the present work is to define a simple and reproducible bioinspired mineralization protocol to precisely nucleate a HA nano-coating on silk organic templates in order to produce a hybrid biomaterial with potential applications in bone TE.

\section{EXPERIMENTAL}

\section{Materials}

The spiders under study were adult females of Cupiennius salei (Keyserling 1877). They were kept in different glass terrarium and fed with a weekly diet of Blaptica dubia or Acheta domestica. All the terrariums were set in the same room with controlled environmental parameters. Each terrarium was provided with a small refuge by considering the need of the spider to allow the animal to feel protected and live without stress, according to the Italian regulation on animal protection and EU Directive 2010/63/EU for animal experiments.

Phosphoric acid $\left(\mathrm{H}_{3} \mathrm{PO}_{4}\right.$, purity $\left.85 \mathrm{wt} \%\right)$ and calcium hydroxide $\left(\mathrm{Ca}[\mathrm{OH}]_{2}\right.$, purity $\left.95 \mathrm{wt} \%\right)$ were purchased from Sigma Aldrich (St. Louis, Missouri, USA). Synthetic HA powder was obtained from Riedel-de Haën (Seelze, Germany). 


\section{Silk Sampling}

Segments of dragline were collected from the spider Cupiennius salei, which produces it continuously during its walking around the terrarium. The dragline is a thread composed by two or three fibers, and it is used by the spiders in order to not fall down from surfaces. ${ }^{31}$ Silk samples were prepared by following the procedure reported by Blackledge et al. ${ }^{32}$ Briefly, specimens were stuck on a plastic holder provided with a window of $1 \times 1 \mathrm{~cm}$ and fixed with a double-sided tape.

\section{Mineralization of Spider Silk}

Each plastic holder containing the silk fibers was fixed on the bottom of a well in a 6-multiwell culture plate (Corning Costar, Corning, New York, USA) with masking tape. A basic suspension and an acidic solution were prepared by, respectively, dispersing $\mathrm{Ca}(\mathrm{OH})_{2}$ and $\mathrm{H}_{3} \mathrm{PO}_{4}$ in milli-Q water (ASTM Type I ultrapure water, arium comfort; Sartorius GmbH, Göttingen, Germany) and kept under magnetic stirring. Reagent solutions with 1, 0.1, and 0.01 M concentration were prepared to perform three different reactions (Figure 1). For the HA deposition (1), used as control, the acidic and the basic solutions were mixed together under gentle magnetic stirring and let to stand for $30 \mathrm{~min}$ to initiate the HA crystal nucleation; the precursor solution was then poured into the well containing the silk fiber. In the biomineralization synthesis (2), silk fiber was covered with the $\mathrm{Ca}(\mathrm{OH})_{2}$ suspension and the phosphoric solution was immediately added dropwise. The reversal biomineralization process (3) was performed by inverting the reagents order compared to process (2), thus dropping the basic suspension over the silk, previously immersed in the acidic solution. All the syntheses were performed at room temperature, and the reagent volume $(5 \mathrm{~mL})$ was calculated to ensure a $\mathrm{Ca} / \mathrm{P}$ ratio of 1.67 , typical of stoichiometric HA. The reaction product was kept under low mechanical shaking at $37 \mathrm{C}$ in incubator (M250-RH, MPM Instruments s.r.1.,

Bernareggio, Italy) for different maturation times (1, 3, or 7 days, Figure 1). The mineralized silk was subsequently removed from the well and washed with milli-Q water for two consecutive times to eliminate any synthesis debris and dried in stove (FP 53, Binder $\mathrm{GmbH}$, Tuttlingen, Germany) at $40 \mathrm{C}$ for $1 \mathrm{~h}$.

\section{Morphological and Chemical Characterization of Mineralized Spider Silks}

Silk surface morphology was examined by scanning electron microscopy (SEM-FEI, Quanta 200, Thermo Fisher Scientific, Watham, Massachusetts, USA) operating at $5 \mathrm{kV}$. The evaluation of the crystal dimension was performed using an image processing software (Fiji, ImageJ). ${ }^{33}$ The elliptical shape of the crystals has been taken into account by measuring both the major and minor axis dimensions, with a minimum number of measurement $n=30$ per each image. Three different images for each mineralization process have been analyzed for the calculation of the average dimensions. Quantitative chemical microanalysis of the mineral phase was performed by energy-dispersive X-ray analysis (EDS-INCA Suite, Oxford Instruments, Abingdon, UK) with an accelerating voltage

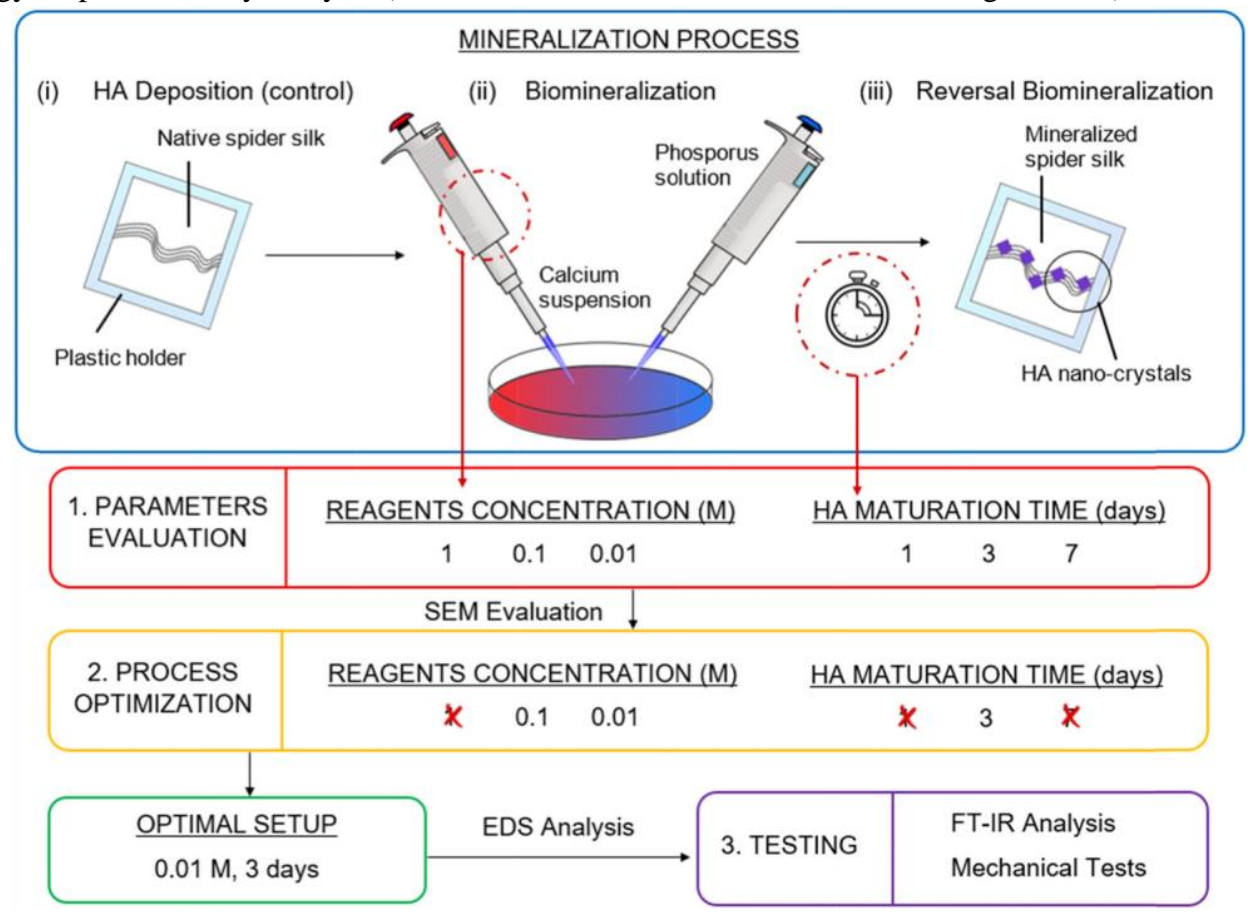

Figure 1. Process overview: parameter evaluation, process optimization, and testing for the three mineralization processes. [Color figure can be viewed at wileyonlinelibrary.com]

of $8 \mathrm{kV}$ and working distance of $8 \mathrm{~mm}$. All samples were coated with a layer of gold (18 nm of thickness, $60 \mathrm{~s}, 20 \mathrm{~mA})$ (Sputter Coater Q150TES, Quorum Technologies Ltd, Laughton, UK). 
Fourier transform infrared spectrometer (FTIR, Nicolet iS5, Thermo Fisher Scientific) equipped with a monolithic diamond for attenuated total reflectance detection [iD7 ATR (attenuated total reflection) Thermo Fisher Scientific] was used to collect mid-IR spectra (400-4000 $\mathrm{cm}^{-1}$, resolution of $4 \mathrm{~cm}^{-1}$ ). Spectral analysis (Omnic Spectra Software, Thermo Fisher Scientific) was performed on (a) mineralized silks, (b) the residues of HA after biomineralization, (c) the raw spider silk, and (d) a commercial HA powder, used as reference material.

The phase analysis of the synthesized HA was conducted by using an X-ray diffractometer (XRD, D8 Advance, Bruker AXS GmbH, Karlsruhe, Germany) equipped with a Lynx-eye positionsensitive detector ( $\mathrm{Cu}$ K $\alpha$ radiation, $\alpha=1.5418 \AA$ ). XRD spectra were recorded at a step size $(2 \theta)$ of 0.02 from 20 to 80 and a scan speed of $1 \mathrm{~s}$.

\section{Mechanical Tests}

Tensile tests were carried out on silks produced with the optimized setup parameters using a nanotensile machine (T150 UTM, Agilent Technologies, Santa Clara, California, USA) with a cell load of $500 \mathrm{mN}$, nominal declared sensitivity of $10 \mathrm{nN}$ for the load in the dynamic configuration. The displacement speed was $10 \mu \mathrm{m} / \mathrm{s}$ with a frequency load of $20 \mathrm{~Hz}$. Before mounting the samples, the number of threads and the diameters of each specimen were measured with an optical microscope in order to compute the area of the total cross section involved. Sample holder was mounted between the clamps to keep the silk fibers parallel to the clamps and cut before the trials. At least 10 samples were tested for each silk type and the mean values ( SD) of strength (MPa), strain at break $(\mathrm{mm} / \mathrm{mm})$, toughness $\left(\mathrm{MJ} / \mathrm{m}^{3}\right)$, and Young's modulus $(\mathrm{GPa})$ were calculated from the stress- strain curves.

\section{Statistical Methods}

Weibull statistics was performed for each set of data to obtain Weibull parameters, namely, scale and shape parameters, through the maximum likelihood method. ${ }^{34}$ Analyses were carried out using Mathematica as processing software. Once the parameters were obtained, their relative probability distributions were plotted.

\section{RESULTS AND DISCUSSION}

In the present study, two bio-inspired mineralization processes were performed to grow HA nano-crystals on native spider draglines and to develop a biomimetic hybrid material suitable for bone tissue regeneration. The process and the experimental parameters were optimized to achieve a controlled HA nucleation and a homogeneous coating. Due to the low availability and the reduced dimensions of the raw material (few $\mu \mathrm{m}$ in diameter), all steps required a fine control during the dragline manipulation. Reagent concentration and HA maturation time were evaluated, since they are known to affect crystal nucleation, size, morphology, orientation, as well as chemical composition and coating homogeneity. ${ }^{33-37}$

\section{Evaluation of the Effect of Reagent Concentration, HA}

Maturation Time, and Mineralization Process on Silk Coating SEM was used to evaluate the coating homogeneity and the HA crystal features, confirming that the HA crystal nucleation depends on the concentration of free ions in the reaction medium and the incubation period. A reagent concentration of $1 \mathrm{M}$ suddenly produced macroscopic HA aggregates on the well bottom after mixing the reagents and resulted in a nucleation of 

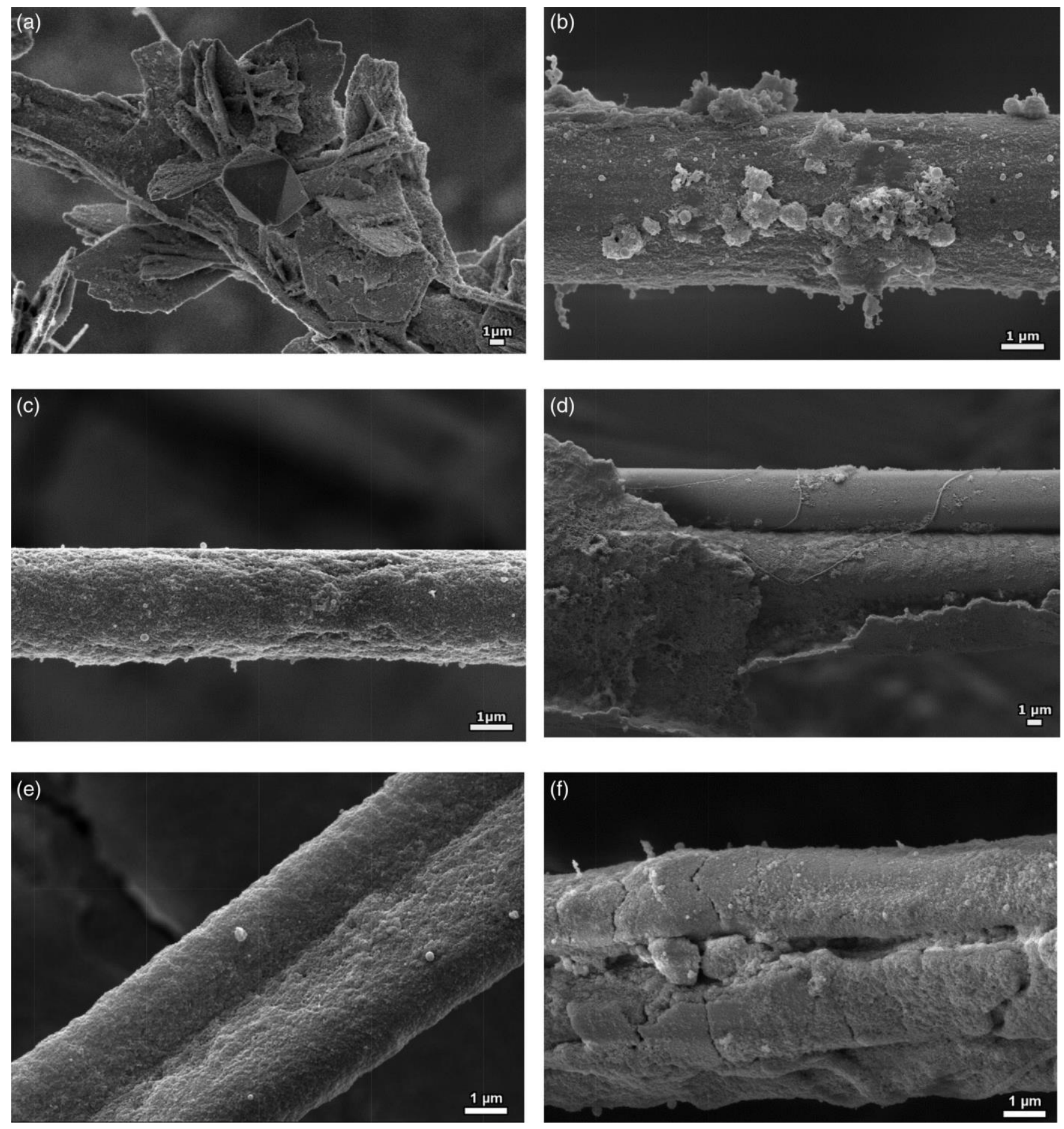

Figure 2. SEM images of spider silks mineralized using different process parameters. Silks mineralized by varying the reagent concentrations: (a) $1 \mathrm{M}$ of reagent concentrations ( 1 day of HA maturation, biomineralization protocol); (b) $0.1 \mathrm{M}$; and (c) $0.01 \mathrm{M}$ ( 3 days of HA maturation time, reversal biomineralization). Silks mineralized by varying the HA maturation time: (d) 1 day (0.1 M of reagent concentrations, biomineralization), (e) 3 days and (f) 7 days

(0.01 $\mathrm{M}$ of reagent concentrations, reversal biomineralization).

nonconfined micrometric calcium phosphate flakes [Figure 2(a)] due to the rapid consumption of calcium and phosphate ions from the reagent solution. ${ }^{38-40}$ The aggregates resulted to be hard to wash and led to a breaking of specimens at any maturation time. Mineralized silks produced with $0.1 \mathrm{M}$ of reagent concentration were characterized by local HA aggregates with a typical "cauliflowerlike" morphology (dimension of a hundred of nanometers to few micrometers) and grape-like aggregates that determine a 
nonhomogeneous morphology [Figure 2(b)]. A reagent concentration of $0.01 \mathrm{M}$ led to a homogeneous formation of HA nano-crystals over the entire fiber length, with crystals length lower than $100 \mathrm{~nm}$ [Figure 2(c)]. Analyzing the effect of different
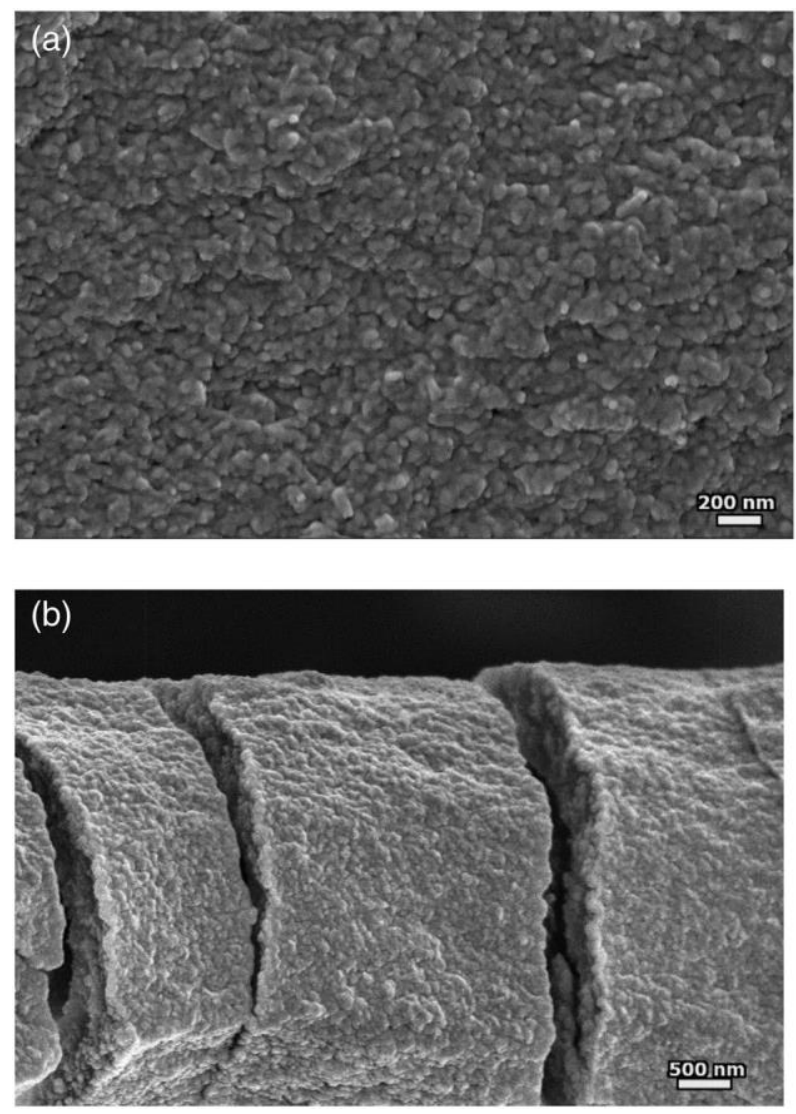

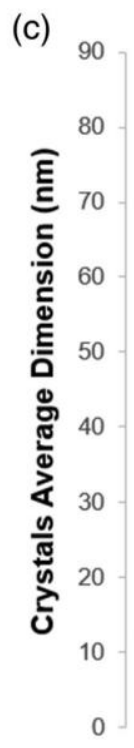

-HA Deposition

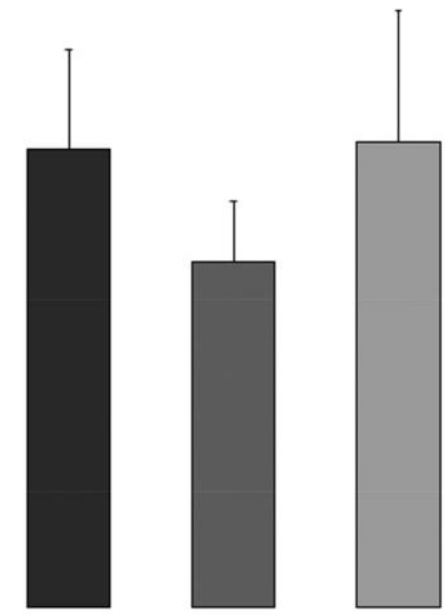

घBiomineralization $\quad$ Reversal Biomineralization

Figure 3. SEM images of mineralized silk (reversal biomineralization, reagent concentrations of $0.01 \mathrm{M}, 3$ days of HA maturation) show (a) the nucleation of a nanometric uniform coating and (b) its thickness in correspondence with the coating fracture. (c) Crystal average dimension has been calculated for the three mineralization processes.

incubation time (1,3, and 7 days), SEM images after 1 day revealed the formation of a discontinuous layer of inorganic phase with partially melted crystals and the presence of micrometric aggregates while large parts of the silks remained completely bare [Figure 2(d)]. Three days resulted to be a sufficient incubation time to obtain a complete and homogeneous apatite layer over the fiber [Figure 2(e)] while an increase of the incubation time up to 7 days caused the growth of clusters of inorganic phases [Figure 2(f)].

Reagent concentrations of 0.1 and $0.01 \mathrm{M}$ and 3 days of samples incubation were thus chosen as suitable variables to have uniform mineralization and minimize the formation of large inorganic

Table I. EDS Results of Ca/P Ratios for Mineralized Spider Silks (Reagent Concentrations of 0.1 and 0.01 M and HA Maturation Time of 3 days)

\begin{tabular}{|c|c|c|}
\hline & $0.1 \mathrm{M}$ & $0.01 \mathrm{M}$ \\
\hline Process & \multicolumn{2}{|c|}{$\mathrm{Ca} / \mathrm{P}$ mean (atomic \%) } \\
\hline HA deposition & 1.690 .12 & 1.680 .30 \\
\hline Biomineralization & 1.810 .16 & 1.780 .30 \\
\hline Reversal biomineralization & 1.630 .23 & 1.560 .12 \\
\hline
\end{tabular}

Concentration

aggregates. The morphological analysis performed on samples treated for 3 days with a $0.01 \mathrm{M}$ reagent concentration showed that the 3 treatments produced a uniform inorganic coating composed of partially fused nano-crystals with rod-like shape and homogeneous thickness of few hundred micrometers [Figure 3(a,b)]. The calculated crystal average dimension resulted to be $6113 \mathrm{~nm}$, $468 \mathrm{~nm}$, 
and $6218 \mathrm{~nm}$ for HA deposition, biomineralization, and reversal biomineralization, respectively, with no statistically significant differences among the three methods [Figure 3(c)].

\section{EDS, FTIR ATR, and XRD Analyses}

Elemental analyses were used to assess the calcium phosphate phase formation for silks processed with reagent concentrations of 0.1 and 0.01 M (3 days of HA maturation) via the three processes (Table I). Results showed that the HA deposition leads to formation of a stoichiometric HA phase, confirmed by a $\mathrm{Ca} / \mathrm{P}$ of about 1.67 for both the reagent concentrations tested while the biomineralization process induces the formation of a mineral phase with a $\mathrm{Ca} / \mathrm{P}$ ratio biased toward the calcium $(\mathrm{Ca} / \mathrm{P}$ higher than 1.67$)$, revealing a preferential and faster binding of $\mathrm{Ca}^{2+}$

ions by the spider draglines. ${ }^{29,41,42}$

Although no significant differences of crystal morphologies and sizes between the biomineralization and the reversal process
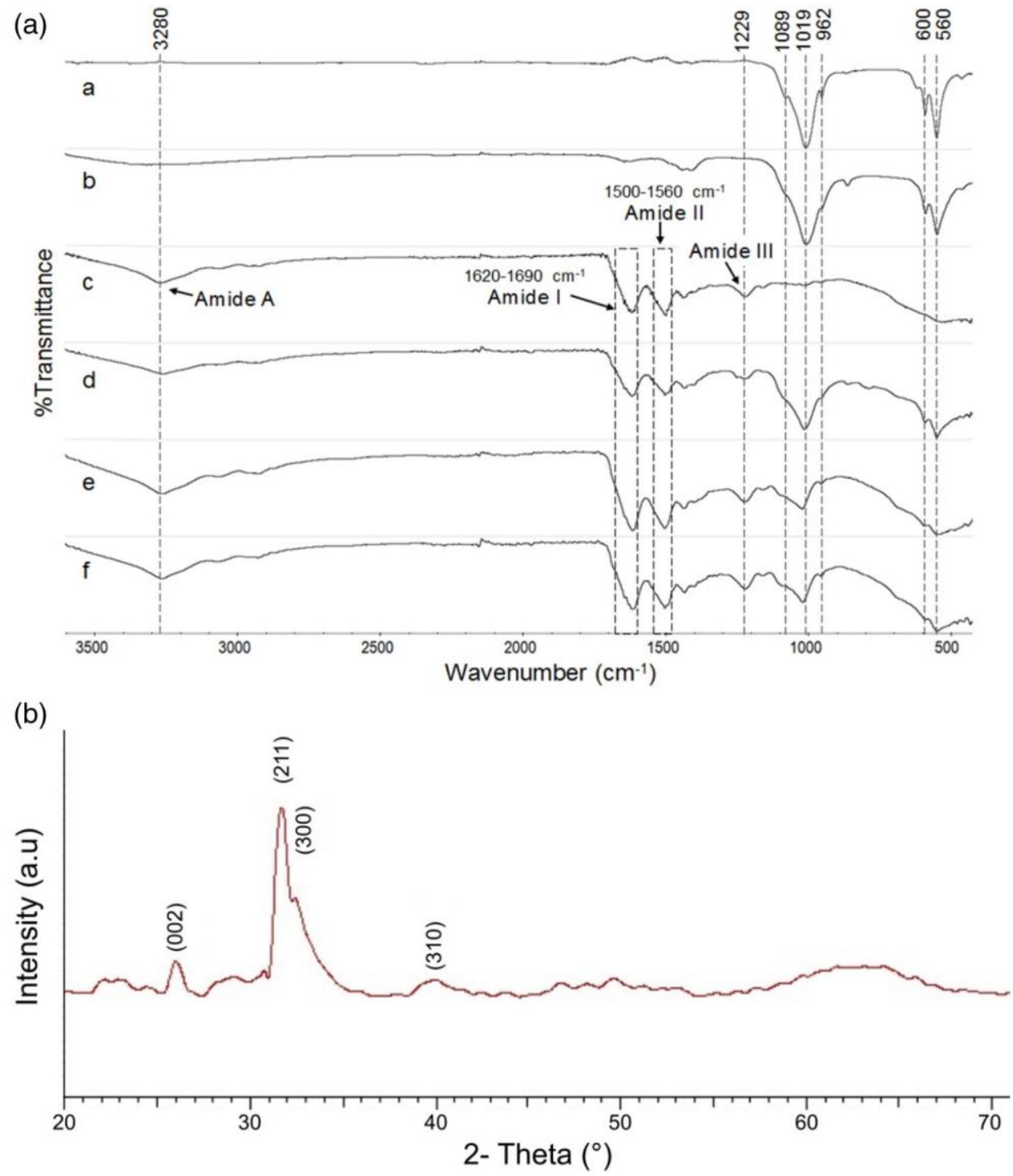

Figure 4. (a) FTIR spectra of (a) commercial HA powder, (b) HA residue of synthesis, (c) raw material (native spider silk dragline), (d) silks mineralized by HA deposition, (e) silks mineralized by biomineralization (neutralization process), and (f) silks mineralized by reversal biomineralization. (b) XRD pattern of HA powder nucleated by biomineralization. [Color figure can be viewed at wileyonlinelibrary.com]

were found (Figure 3), data from EDS analyses revealed that the reversal process entails a reduction of the calcium uptake from the silk, leading to a $\mathrm{Ca} / \mathrm{P}$ ratio typical of nonstoichiometric, biomimetic calcium-deficient $\mathrm{HA}$ (with a $\mathrm{Ca} / \mathrm{P}$ ratio of $1.50-1.67){ }^{39,43}$

FTIR ATR spectra of both commercial HA powder (control) and HA residues from the biomineralization process [Figure 4 (a), spectra $\mathrm{a}$ and $\mathrm{b}$ ] showed peaks at $600 \mathrm{~cm}^{-1}$,typical of $\mathrm{PO}_{4}{ }^{3-}$ bending mode, and peaks at about 960,1000 , and $1090 \mathrm{~cm}^{-1}$, assigned to the $\mathrm{PO}_{4}{ }^{3-}$ stretching mode, confirming the highly crystalline HA phase of the synthesis residues. The native spider spectrum [spectrum $\mathrm{c}$ in Figure 4(a)] revealed the presence of amide III $\left(1230 \mathrm{~cm}^{-1}\right)$, amide II $\left(1500-1560 \mathrm{~cm}^{-1}\right)$, amide I $\left(1620-1690 \mathrm{~cm}^{-1}\right)$, and amide A (at 
about $3280 \mathrm{~cm}^{-1}$ ). The presence of the characteristic apatite peaks in the mineralized silk spectra proved that a successful nucleation of HA occurred for all the mineralization processes ${ }^{44-46}$ [Figure 4(a), spectra d, e and f]. According to the EDS analyses, the biomineralization and reversal biomineralization processes led to a broadening of the apatite peaks compared to both HA residues and HA deposition spectra, suggesting the role of spider silk as a template for the growth of a biomimetic low-crystalline HA coating. The remaining bands are ascribed to spider silk, as shown by the raw material spectrum. ${ }^{47-49}$

To confirm the chemical composition of the mineral phase, XRD analysis was performed on the reaction powders obtained from the biomineralization process $(0.01 \mathrm{M}$ reagent concentration) after 1 day of maturation. The XRD pattern exhibited a scarcely crystalline profile of the mineral phase, with broad undulations and few discrete peaks, in good agreement with EDS and FTIR results [Figure 4(b)]. The diffraction peaks resulted to be typical of a calcium phosphate HA according to the main lattice reflections of the JCPDSICDD file (Card \# 09-0432).

Table II. Measured and Computed Mechanical Properties of Raw Spider Silk, Supercontracted Spider Silk, and Mineralized Spider Silk (0.01 M of Reagent Concentrations, 3 days of HA Maturation)

\begin{tabular}{|c|c|c|c|c|c|c|}
\hline Process & $\begin{array}{l}\text { Strength } \\
(\mathrm{MPa})\end{array}$ & $\begin{array}{l}\text { Strain at break } \\
(\mathrm{mm} / \mathrm{mm})\end{array}$ & $\begin{array}{l}\text { Toughness } \\
\left(\mathrm{MJ} / \mathrm{m}^{3}\right)\end{array}$ & $\begin{array}{l}\text { Young's modulus } \\
\text { (GPa) }\end{array}$ & $\begin{array}{l}\text { Weibull scale parameter } \\
(\mathrm{MPa})\end{array}$ & $\begin{array}{l}\text { Weibull shape } \\
\text { parameter }\end{array}$ \\
\hline Raw material & 580520 & 0.270 .03 & 110120 & 8.57 .1 & 620 & 1.2 \\
\hline Supercontracted & 190190 & 0.410 .25 & 5759 & 2.94 .6 & 162 & 1.4 \\
\hline HA deposition & 160100 & 0.320 .17 & 3823 & 2.51 .7 & 156 & 1.4 \\
\hline Biomineralization & 150120 & $0.44 \quad 0.27$ & 5749 & 4.62 .7 & 161 & 1.4 \\
\hline $\begin{array}{l}\text { Reversal } \\
\text { biomineralization }\end{array}$ & 12090 & 0.450 .25 & 4139 & 2.31 .5 & 135 & 1.5 \\
\hline
\end{tabular}

Mechanical Properties

contribute in reducing the strength because of an increase of the Uniaxial tensile tests were performed on specimens from all fiber diameter compared to the raw material. groups (silks treated by HA deposition, biomineralization, and

Supercontraction is also responsible for the increase of strain at

reversal biomineralization), mineralized with a reagent concentra- break and the reduction of Young's modulus. This is due to the tion of $0.01 \mathrm{M}$ and 3 days of HA maturation. Raw material fact that during hydrogen bond annihilation, there is an increase (untreated spider silk) and supercontracted silk, obtained by of entropic elasticity, which increases the tangle status of the molmaintaining the samples in wet conditions overnight at room ${ }_{55,56}$ ecules. This trend was similar for HA deposition and reversal temperature and $100 \%$ of saturated humidity, were used as conbiomineralization. In fact, silk shrinking determines the forma-

trols. The data revealed that mineralization of spider silks leads tion of more elastic fibers 57 while apatite is a ceramic material to a reduction of the strength, toughness, and Young's modulus characterized by a brittle mechanical behavior, with low tough-

and to an increase of strain at break compared to the raw mate- ness and low resistance to load bearing in comparison with flexirial, a behavior that can be mainly attributed to the silk super- $58-60$

contraction (Table II, Figure 5).

ble materials.

The strength of silks, that is, the stress just before thread's frac-

ture, resulted in a reduction from $580520 \mathrm{MPa}$ of the

The analysis of the Weibull statistics shown that the mineraliza-

tion process leads to a homogenization of the fracture behavior [-

untreated silk to less than $200 \mathrm{MPa}$ for supercontracted and min-

Figure 5(b)] that could be correlated to the recovery of

fibers

after the mineralization process. Shape parameters between eralized silks, with similar values for the three mineralization pro-

1.4 and 1.5 were obtained for all the supercontracted and minercesses. The interaction with water is responsible for silk alized samples, with a narrower probability distribution comsupercontraction, a phenomenon that affects the morphological,

physical, and mechanical properties of spider silk,50-52 with a pared to the raw material, so the fracture behavior is expected to be more deterministic. The reduction of the Weibull scale param-

reduction of strength caused probably by the annihilation and eter indicates a decrease in strength according to the analysis of reorganization of silk hydrogen bonds in contact with water mol-53,54 the stress-strain curves. Namely, the mineralization process ecules. The presence of an inorganic coating could also 

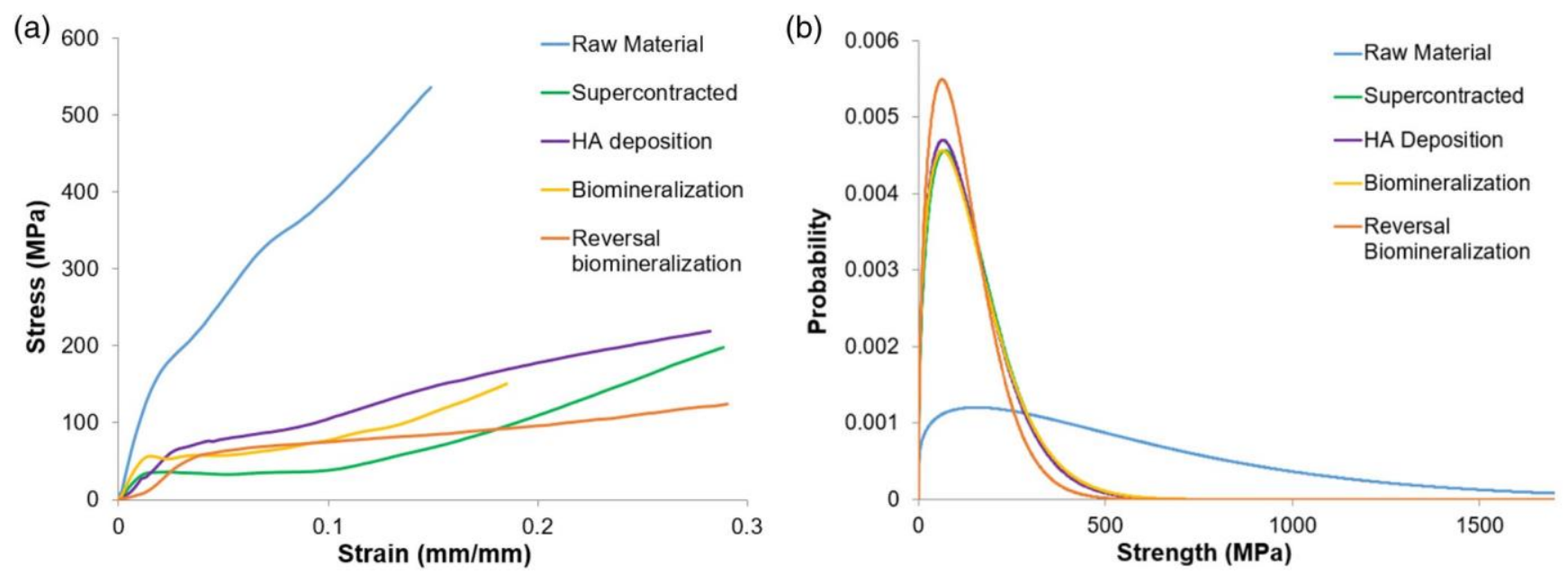

Figure 5. (a) Stress-strain curves and (b) Weibull probability distribution of the raw material, supercontracted silk, and mineralized spider silks. [Color figure can be viewed at wileyonlinelibrary.com]

and $135 \mathrm{MPa}$ for the HA deposition, biomineralization, and reversal biomineralization, respectively.

Although the supercontraction affects notably the mechanical behavior of native silk, mineralized silks still present performances comparable to many natural and artificial fibers with remarkable mechanical properties. ${ }^{64}$ Furthermore, the hybrid composite shows mechanical properties comparable to that of natural bone in terms of strength and elastic modulus ${ }^{65}$ and superior to many natural and synthetic biomaterials used in bone

TE. 66,67

\section{CONCLUSION}

This study demonstrated that native spider silk can be coated with a nanostructured mineral layer by tuning neutralization process parameters to get a reproducible and effective surface functionalization. Two different bio-inspired neutralization processes were performed, and the mineralized silks were compared to raw material, supercontracted silks, and samples produced by simple HA deposition. Morphological analyses revealed that the use of $0.01 \mathrm{M}$ of reagent concentration and 3 days of apatite maturation led to a homogeneous and thin coating of HA nano-crystals. FTIR and XRD spectra confirmed the nucleation of a lowcrystalline HA phase onto spider silk, and data from EDS demonstrated that the reversal biomineralization conveyed the nucleation of biomimetic, poorly crystalline, and nonstoichiometric HA. Mechanical results revealed that silk supercontraction is mainly responsible for a reduction in elasticity, strength, and toughness of mineralized silks, nevertheless ensuring outstanding mechanical properties, comparable to those of human bone.

In summary, the fine tuning of biomineralization parameters led to the controlled nucleation of biomimetic HA onto native spider silk while maintaining good mechanical performances, making this hybrid biomaterial interesting for bone TE applications. Biological in vitro tests and the design of scaffolds that incorporate biomineralized spider silk for enhancing mechanical properties while providing a biomimetic interface will be pursued in future studies.

\section{ACKNOWLEDGMENTS}

This work was supported by regional funding from NanoCoatings project (POR-FESR 2014-2020, grant number D32I16000040009) as well as by the National Research Council of Italy (ISTEC-CNR). N.M.P. is supported by the European Commission under the Graphene Flagship Core 2 grant number 785219 (WP14 "Composites") and FET Proactive "Neurofibres" grant number 732344 as well as by the Italian Ministry of Education, University and Research (MIUR) under the "Departments of Excellence" grant L.232/2016 and ARS01-01384-PROSCAN and the PRIN-20177TTP3S. 


\section{REFERENCES}

1. Kaplan, D. L.; Lombardi, S. J.; Muller, W. S.; Fossey, S. A.Biomaterials; Palgrave Macmillan UK: London, UK, 1991 ; p. 53.

2. Altman, G. H.; Diaz, F.; Jakuba, C.; Calabro, T.; Horan, R. L.; Chen, J.; Lu, H.; Richmond, J.; Kaplan, D. L. Biomaterials. 2003, 24,401 .

3. Mirahmadi, F.; Tafazzoli-Shadpour, M.; Shokrgozar, M. A.;Bonakdar, S. Mater. Sci. Eng. C. 2013, 33 , 4786.

4. Eisoldt, L.; Smith, A.; Scheibel, T. Mater. Today. 2011, 14, 80.

5. Mackenzie, D. Med. Hist. 1973, 17, 158.

6. Dhandayuthapani, B.; Yoshida, Y.; Maekawa, T.; Kumar, D. S. Int. J. Polym. Sci. 2011, 2011, 290602.

7. Kim, U.-J.; Park, J.; Li, C.; Jin, H.-J.; Valluzzi, R.; Kaplan, D. L. Biomacromolecules. 2004, 5, 786.

8. Vepari, C.; Kaplan, D. L. Prog. Polym. Sci. 2007, 32, 991.

9. Kundu, J.; Chung, Y.-I.; Kim, Y. H.; Tae, G.; Kundu, S. C.Int. J. Pharm. 2010, 388, 242.

10. Elsner, M. B.; Herold, H. M.; Müller-Herrmann, S.; Bargel, H.; Scheibel, T. Biomater. Sci. 2015, 3, 543.

11. Jao, D.; Mou, X.; Hu, X. J. Funct. Biomater. 2016, 7, 22.

12. Kim, H. J.; Kim, U.-J.; Kim, H. S.; Li, C.; Wada, M.;Leisk, G. G.; Kaplan, D. L. Bone. 2008, 42, 1226.

13. Shi, P.; Teh, T. K. H.; Toh, S. L.; Goh, J. C. H. Biomaterials. 2013, 34, 5947.

14. Rising, A.; Widhe, M.; Johansson, J. Cell. Mol. Life Sci. 2011, 68, 169.

15. Cranford, S. W.; Tarakanova, A.; Pugno, N. M.; Buehler, M. J. Nature. 2012, 482, 72.

16. Yang, Y.; Greco, G.; Maniglio, D.; Migliaresi, C.; Pugno, N.;Motta, A. Mater. Sci. Eng. C. 2019, $107,110197$.

17. Schacht, K.; Scheibel, T. Curr. Opin. Biotechnol. 2014, 29, 62.

18. Hardy, J. G.; Torres-Rendon, J. G.; Leal-Egaña, A.; Walther, A.; Schlaad, H.; Cölfen, H.; Scheibel, T. R. Materials (Basel). 2016, 9,560 .

19. Rising, A.; Johansson, J. Nat. Chem. Biol. 2015, 11, 309.

20. Andersson, M.; Jia, Q.; Abella, A.; Lee, X.-Y.; Landreh, M.;Purhonen, P.; Hebert, H.; Tenje, M.; Robinson, C. V.; Meng, Q. Nat. Chem. Biol. 2017, 13, 262.

21. Bhattacharjee, P.; Kundu, B.; Naskar, D.; Kim, H.-W.; Maiti, T. K.; Bhattacharya, D.; Kundu, S. C. Acta Biomater. $2017,63,1$.

22. Yang, M.; He, W.; Shuai, Y.; Min, S.; Zhu, L. J. Polym. Sci. Part B Polym. Phys. 2013, $51,742$.

23. Thavornyutikarn, $\quad$ B.; $\quad$ Chantarapanich, N.; Sitthiseripratip, K.; Thouas, G.; Chen, Q. Prog. Biomater. $2014,3,61$.

24. Tampieri, A.; Celotti, G.; Landi, E.; Sandri, M.; Roveri, N.;Falini, G. J. Biomed. Mater. Res. Part A. $2003,67,618$.

25. Stevens, B.; Yang, Y.; Mohandas, A.; Stucker, B.; Nguyen, K. T. J. Biomed. Mater. Res. Part B Appl. Biomater. $2008,85,573$.

26. Palmer, L. C.; Newcomb, C. J.; Kaltz, S. R.; Spoerke, E. D.;Stupp, S. I. Chem. Rev. 2008, 108, 4754.

27. Stupp, S. I.; Ciegler, G. W. J. Biomed. Mater. Res. Part A. 1992, 26, 169.

28. Nayak, A. K. Int. J. ChemTech Res. 2010, 2, 903.

29. Cao, B.; Mao, C. Langmuir. 2007, 23, 10701.

30. Dmitrovic, S.; Jokic, B.; Prekajski, M.; Pantic, J.; Zmejkoski, D.; Zarubica, A.; Matovic, B. Process. Appl. Ceram. $2016,10,37$.

31. Greco, G.; Pantano, M. F.; Mazzolai, B.; Pugno, N. M. Sci. Rep. 2019, 9, 5776.

32. Blackledge, T. A.; Swindeman, J. E.; Hayashi, C. Y. J. Exp. Biol. 2005, 208, 1937.

33. Schindelin, J.; Arganda-Carreras, I.; Frise, E.; Kaynig, V.;Longair, M.; Pietzsch, T.; Preibisch, S.; Rueden, C.; Saalfeld, S.; Schmid, B.; Tinevez, J. Y.; White, D. J.; Hartenstein, V.; Eliceiri, K.; Tomancak, P.; Cardona, A. Nat. Methods. 2012,9 , 676.

34. Peterlik, H. J. Mater. Sci. 1972, 1995, 30.

35. Cazalbou, S.; Combes, C.; Eichert, D.; Rey, C.; Glimcher, M. J. J. Bone Miner. Metab. 2004, $22,310$.

36. Jongprateep, O.; Nueangjumnong, C.. In Proceedings of theInternational Conference on Advanced Materials, Structures and Mechanical Engineering; 2016; pp 251.

37. Nancollas, G. H.; Mohan, M. S. Arch. Oral Biol. 1970, 15, 731.

38. Jin, Y.; Kundu, B.; Cai, Y.; Kundu, S. C.; Yao, J. Colloids Surfaces B Biointerfaces. 2015, 134, 339. 
39. Dorozhkin, S. V. Materials (Basel). 2009, 2, 1975.

40. Lluch, A. V.; Ferrer, G. G.; Pradas, M. M. Colloids Surfaces B Biointerfaces. 2009, 70, 218.

41. Thiel, B. L.; Kunkel, D. D.; Viney, C. Biopolymers. 1994, 34, 1089.

42. Kino, R.; Ikoma, T.; Monkawa, A.; Yunoki, S.; Munekata, M.; Tanaka, J.; Asakura, T. J. Appl. Polym. Sci. $2006,99,2822$.

43. Bohner, M. Injury. 2000, 31, D37.

44. Hooshmand, T.; Abrishamchian, A.; Najafi, F.; Mohammadi, M.; Najafi, H.; Tahriri, M. J. Compos. Mater. $2014,48,483$.

45. Gheisari, H.; Karamian, E.; Abdellahi, M. Ceram. Int. 2015, 41, 5967.

46. Kede, M. L. F. M.; Mavropoulos, E.; da Rocha, N. C. C.;Costa, A. M.; da Silva, M. H. P.; Moreira, J. C.; Rossi, A. M. Surf. Coatings Technol. 2012, 206, 2810.

47. Papadopoulos, P.; Sölter, J.; Kremer, F. Eur. Phys. J. E. 2007, 24, 193.

48. Dong, Z.; Lewis, R. V.; Middaugh, C. R. Arch. Biochem. Biophys. 1991, 284, 53.

49. Slotta, U.; Tammer, M.; Kremer, F.; Koelsch, P.; Scheibel, T. Supramol. Chem. 2006, 18, 465.

50. Saravanan, D. J. Text. Apparel, Technol. Manag. 2006, 5, 1.

51. Work, R. W. Text. Res. J. 1977, 47, 650.

52. Boutry, C.; Blackledge, T. A. J. Exp. Biol. 2013, 216, 3606.

53. Tokareva, O.; Jacobsen, M.; Buehler, M.; Wong, J.; Kaplan, D. L. Acta Biomater. 2014, 10, 1612.

54. Nova, A.; Keten, S.; Pugno, N. M.; Redaelli, A.; Buehler, M. J. Nano Lett. 2010, 10, 2626.

55. Berardo, A.; Pantano, M. F.; Pugno, N. M. Interface Focus. 2016, 6, 20150060.

56. Liu, Y.; Shao, Z.; Vollrath, F. Nat. Mater. 2005, 4, 901.

57. Bell, F. I.; McEwen, I. J.; Viney, C. Nature. 2002, 416, 37.

58. Yan, W.-Q.; Nakamura, T.; Kawanabe, K.; Nishigochi, S.;Oka, M.; Kokubo, T. Biomaterials. 1997, $18,1185$.

59. Johnson, A. J. W.; Herschler, B. A. Acta Biomater. 2011, 7, 16.

60. Ramesh, N.; Moratti, S. C.; Dias, G. J. J. Biomed. Mater. Res. Part B Appl. Biomater. 2017, 106, 2046.

61. Pérez-Rigueiro, J.; Elices, M.; Guinea, G. V. Polymer (Guildf). 2003, 44, 3733.

62. Elices, M.; Plaza, G. R.; Pérez-Rigueiro, J.; Guinea, G. V. J. Mech. Behav. Biomed. Mater. 2011, 4, 658.

63. Agnarsson, I.; Dhinojwala, A.; Sahni, V.; Blackledge, T. A. J. Exp. Biol. 2009, 212, 1990.

64. Gosline, J.; Lillie, M.; Carrington, E.; Guerette, P.; Ortlepp, C.; Savage, K. Philos. Trans. R. Soc. B Biol. Sci. 2002, $357,121$.

65. Mow, V. C.; Huiskes, R. Basic Orthopaedic Biomechanics \& Mechano-Biology; Lippincott Williams \& Wilkins: Philadelphia, PA, USA, 2005.

66. Sabir, M. I.; Xu, X.; Li, L. J. Mater. Sci. 2009, 44, 5713.

67. Woodard, J. R.; Hilldore, A. J.; Lan, S. K.; Park, C. J.;Morgan, A. W.; Eurell, J. A. C.; Clark, S. G.; Wheeler, M. B.; Jamison, R. D.; Wagoner Johnson, A. J. Biomaterials. 2007, 28, 45. 